\title{
Corosolic acid induces apoptotic cell death in HCT116 human colon cancer cells through a caspase-dependent pathway
}

\author{
BOKYUNG SUNG ${ }^{1}$, YONG JUNG KANG ${ }^{1}$, DONG HWAN KIM ${ }^{1}$, SEONG YEON HWANG ${ }^{1}$, YUJIN LEE ${ }^{1}$, \\ MINJEONG KIM ${ }^{1}$, JEONG-HYUN YOON ${ }^{1}$, CHEOL MIN KIM ${ }^{2,3}$, HAE YOUNG CHUNG ${ }^{1}$ and NAM DEUK KIM ${ }^{1,2}$ \\ ${ }^{1}$ Department of Pharmacy, Molecular Inflammation Research Center for Aging Intervention (MRCA) and \\ ${ }^{2}$ Research Center for Anti-Aging Technology Development, Pusan National University, Busan 609-735; \\ ${ }^{3}$ Department of Biochemistry, Pusan National University School of Medicine, \\ Yangsan 626-770, Republic of Korea
}

Received November 27, 2013; Accepted January 21, 2014

DOI: $10.3892 /$ ijmm.2014.1639

\begin{abstract}
Corosolic acid (CA), a pentacyclic triterpene isolated from Lagerstroemia speciosa L. (also known as Banaba), has been shown to exhibit anticancer properties in various cancer cell lines. However, the anticancer activity of CA on human colorectal cancer cells and the underlying mechanisms remain to be elucidated. In this study, we investigated the effects of CA on cell viability and apoptosis in HCT116 human colon cancer cells. CA dose-dependently inhibited the viability of HCT116 cells. The typical hallmarks of apoptosis, such as chromatin condensation, a sub-G1 peak and phosphatidylserine externalization were detected by Hoechst 33342 staining, flow cytometry and Annexin V staining following treatment with CA. Western blot analysis revealed that CA induced a decrease in the levels of procaspase- $8,-9$ and -3 and the cleavage of poly(ADP-ribose) polymerase (PARP). The apoptotic cell death induced by CA was accompanied by the activation of caspase- $8,-9$ and -3 , which was completely abrogated by the pan-caspase inhibitor, z-VAD-FMK. Furthermore, CA upregulated the levels of pro-apoptotic proteins, such as Bax, Fas and FasL and downregulated the levels of anti-apoptotic proteins, such as Bcl-2 and survivin. Taken together, our data provide insight into the molecular mechanisms of CA-induced apoptosis in colorectal cancer (CRC), rendering this compound a potential anticancer agent for the treatment of CRC.
\end{abstract}

\section{Introduction}

According to recent data, the number of worldwide cancer cases is determined to increase by $75 \%$ over the next two

Correspondence to: Dr Nam Deuk Kim, Department of Pharmacy, Molecular Inflammation Research Center for Aging Intervention, Pusan National University, 63 Beon-gil 2, Busandaehag-ro, Geumjeong-gu, Busan 609-735, Republic of Korea

E-mail: nadkim@pusan.ac.kr

Key words: corosolic acid, colorectal cancer cells, apoptosis, caspase decades (1). In Korea, the incidence of cancer cases has shown an annual increase of $3.3 \%$ from 1999 to 2010. Notably, colorectal cancer (CRC) incidence and mortality has been increasing rapidly in Korea over the past few decades. CRC is the third most common cancer with age-standardized incidences of 48.6/100,000 individuals for men and 25.3/100,000 individuals for women in 2010; since then, these incidences have increased by $5.9 \%$ year in both genders (2). Surgery was initially seen as a curative treatment and has now became a conventional therapy for CRC. However, the recurrence rate is as high as $50 \%$ for patients treated with conventional therapy and this is a major issue. Chemotherapeutic regimens, such as FOLFOX [5-fluorouracil (FU) + oxaliplatin + leucovorin] or FOLFIRI (5-FU + leucovorin + irinotecan), neoadjuvant chemotherapy with bevacizumab, an antibody that targets vascular endothelial growth factor, and/or cetuximab, an antibody that targets epidermal growth factor receptor, have been developed as a strategy to combat the recurrence of CRC (3). In spite of these combinations of multiple chemotherapeutic agents, patients develop resistance to such treatments, thus novel strategies to replace or complement current therapies are urgently required.

Apoptosis, the major form of cell suicide, is critical to various physiological processes and the maintenance of homeostasis in multicellular organisms. Hence, it is clear that the dysregulation of apoptosis plays an important role in the pathogenesis of several of human diseases, including cancer (4). Over the years, accumulating evidence clearly indicates that anticancer drugs are able to induce apoptosis and that this process is involved in the mediation of their cytotoxic effects. Furthermore, the selective regulation of the apoptotic pathway in cancer cells, and not in normal cells has been the goal of cancer researchers (5).

Over the past few decades, agents derived from medicinal plants have gained a great deal of attention from researchers and clinicians due to their safety, efficacy and availability. In addition, secondary metabolites and structural derivatives from natural sources have been applied towards treating cancer for the past five decades. At least $40 \%$ of all available anticancer drugs between 1940 and 2002 have originated from natural sources or mimics of natural agents (6). Although a 
number of natural agents have shown an ability to prevent and treat cancer, their molecular mechanisms of action have been poorly defined. One such agent is corosolic acid (CA), which was originally isolated from the fruits of Crataegus pinnatifida var. psilosa (7). CA (Fig. 1A) is an ursane-type pentacyclic triterpene, which exists in abundance in the plant kingdom. It has been found in various plants, including Schisandra chinensis (8), Eriobotrya japonica (known as loquat) (9), Lagerstroemia speciosa L. (known as Banaba) (10), Orthosiphon stamineus (11) and Weigela subsessilis (12). CA has been shown to exert numerous biological activities, such as anti-diabetic $(10,13)$, antioxidant (14), anti-atherosclerotic (15), cholesterol-reducing $(16)$, anti-inflammatory $(17,18)$ and anticancer activities $(9,12,19-23)$. Previous studies have reported that CA suppresses the proliferation of a wide variety of tumor cells, including sarcoma (20), glioblastoma (22), osteosarcoma (21), leukemia $(7,9)$, as well as gastric $(12,24)$, cervical (23) and lung cancer cells (19).

The mechanisms through which CA exerts these effects are not yet fully understood. However, this triterpene has been known to target numerous cell signaling molecules, such as nuclear factor- $\kappa \mathrm{B}(15)$, signal transducer and activator of transcription-3 (22), AMP-activated protein kinase-mammalian target of rapamycin (12), epidermal growth factor receptor 2/ neu (24), protein kinase C (7), GLUT4 (25), glycogen phosphorylase (26), phosphatidylinositol 3-kinase (27), as well as caspases (21).

Although CA has been shown to induce apoptosis, and suppress cancer cell growth and metastasis in several human cancer cell lines $(7,20-22,24)$, its potential anticancer effects and its mechanisms of action in CRC remain unelucidated. Therefore, in the current study, we investigated whether CA induces apoptosis in colon cancer cells. To our knowledge, our results indicate for the first time that CA exerts anticancer effects through the suppression of cell proliferation and the induction of apoptosis in CRC.

\section{Materials and methods}

Chemicals. CA was purchased from LKT Laboratories (St. Paul, MN, USA). Oleanolic acid (OA) was obtained from Cayman Chemical Co. (Ann Arbor, MI, USA) and ursolic acid (UA) was from Sigma-Aldrich (St. Louis, MO, USA). A $50 \mathrm{mM}$ solution of the triterpenoids was prepared in dimethyl sulfoxide and then diluted as needed in cell culture medium. 3-(4,5-Dimethylthiazol-2-yl)-2,5-dipheny tetrazolium bromide (MTT) was obtained from Amresco (Solon, OH, USA). Various primary antibodies and z-VAD-FMK, a broad spectrum caspase inhibitor, were purchased from Santa Cruz Biotechnology, Inc. (Santa Cruz, CA, USA). Mouse monoclonal antibody against $\beta$-actin and Hoechst 33342 were obtained from Sigma-Aldrich. RPMI-1640, fetal bovine serum (FBS), and penicillin-streptomycin were purchased from HyClone (Logan, UT, USA).

Cell culture and cell viability assay. The human CRC cell line, HCT116, which was obtained from the American Type Culture Collection (Manassas, VA, USA), was maintained at $37^{\circ} \mathrm{C}$ in a humidified condition of $95 \%$ air and $5 \% \mathrm{CO}_{2}$ in RPMI-1640 medium supplemented with $10 \%$ FBS and $1 \%(\mathrm{v} / \mathrm{v})$ penicillin- streptomycin. Cell viability was measured using MTT, which is based on the conversion of MTT to MTT-formazan by mitochondrial enzymes.

Nuclear staining with Hoechst 33342. The control and treated cells were washed with phosphate-buffered saline (PBS) and stained with $4 \mu \mathrm{g} / \mathrm{ml}$ Hoechst 33342 for $20 \mathrm{~min}$ at room temperature. Subsequently, the cells were washed with PBS and observed under a fluorescence microscope.

Western blot analysis. To determine the levels of protein expression, we prepared cell extracts and performed western blot analysis. In brief, cell extracts containing equal amounts of proteins were subjected to sodium dodecyl sulfate-polyacrylamide gel electrophoresis (SDS-PAGE) and transferred onto PVDF membranes. The membranes were probed with the desired primary antibodies and then with horseradish peroxidase-conjugated secondary antibody. Signals were detected by enhanced chemiluminescence (ECL) reagent (GE Healthcare, Piscataway, NJ, USA).

Cell cycle analysis. In order to measure the number of cells in the sub-G1 phase, cell cycle analysis was performed. The cells were seeded in 6 -well plates at $3 \times 10^{5}$ cells/well and allowed to attach overnight. The cells were then treated with various concentrations of CA for a period of $24 \mathrm{~h}$, trypsinized, washed with PBS and then fixed in $70 \%$ ethanol at $-20^{\circ} \mathrm{C}$ overnight. Prior to analyses, the cells were washed with PBS, suspended in cold propidium iodide (PI; Sigma-Aldrich) solution $(50 \mu / \mathrm{ml}$ in PBS), and incubated at room temperature in the dark for $30 \mathrm{~min}$. Flow cytometry was then performed using a Cytomic FC500 flow cytometer (Beckman Coulter, Istanbul, Turkey).

Annexin V/PI staining. In order to determine the number of apoptotic cells following treatment with $\mathrm{CA}$, and double staining with Annexin V and PI was conducted using the BD Pharmingen FITC Annexin V Apoptosis Detection kit (BD Biosciences, San Diego, CA, USA) according to the manufacturer's instructions. Flow cytometric analyses were performed on a Cytomic FC500 flow cytometer (Beckman Coulter).

Caspase activity. To determine the effects of CA on caspasemediated cell death, a caspase activity assay using synthetic tetrapeptides [Asp-Glu-Val-Asp (DEAD) for caspase-3; Ile-Glu-Thr-Asp (IETD) for caspase-8; and Leu-Glu-His-Asp (LEHD) for caspase-9] labeled with p-nitroaniline (pNA) as the substrate was conducted in accordance with manufacturer's instructions (R\&D Systems, Minneapolis, MN, USA).

Statistical analysis. Data are presented as the means \pm standard deviation (SD). Statistical analysis was carried out with the use of a two-tailed unpaired Student's t-test. Values of ${ }^{*} \mathrm{P}<0.05,{ }^{* *} \mathrm{P}<0.01$ and ${ }^{* * *} \mathrm{P}<0.001$ were considered to indicate statistically significant differences.

\section{Results}

We investigated the effects of CA on apoptosis and on various regulatory genes involved in apoptosis and the cell cycle in 
A
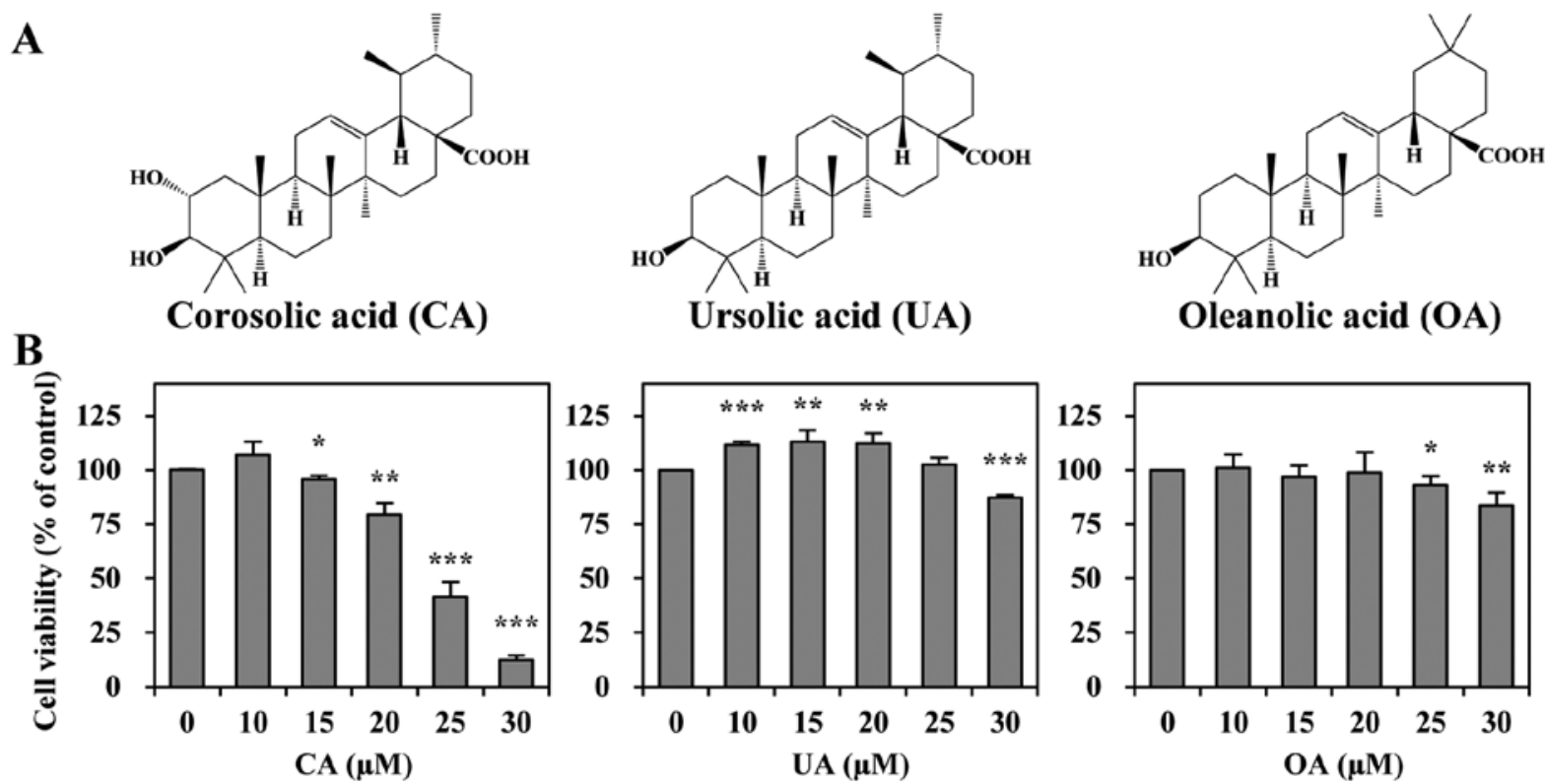

Ursolic acid (UA)

Oleanolic acid (OA)
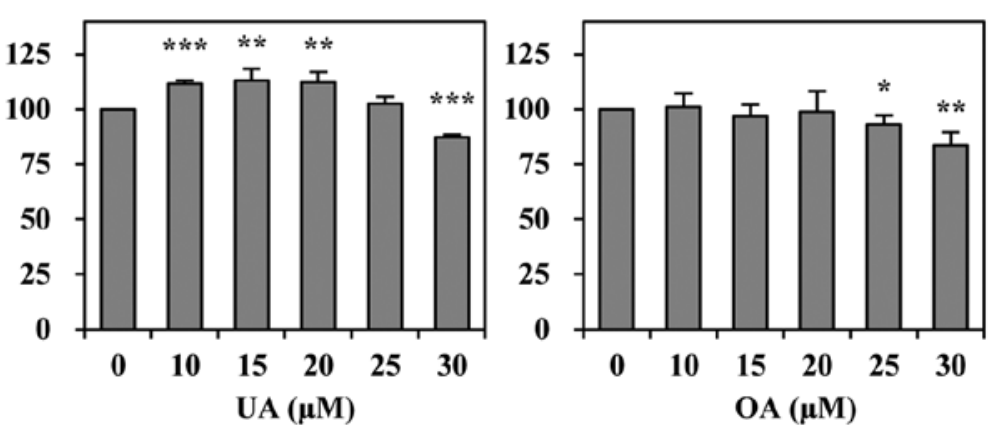

Figure 1. Effects of corosolic acid (CA) and its structural derivatives on the viability of the human colorectal cancer cell line, HCT116. (A) Chemical structures of CA, ursolic acid (UA) and oleanolic acid (OA). (B) Cells were treated with various concentrations of triterpenoids for 24 h, and cell viability was determined by MTT assay. Results were expressed as a percentage of untreated controls \pm standard deviation (SD) obtained from three separate experiments. ${ }^{*} \mathrm{P}<0.05$, ${ }^{* *} \mathrm{P}<0.01$ and ${ }^{* * *} \mathrm{P}<0.001$ compared with the control (untreated) group.

CRC cells. In addition, we also evaluated the role of caspases in CA-induced apoptosis.

CA exerts a potent cytotoxic effect compared to its structural derivatives. We investigated whether $\mathrm{CA}$ and its structural analogues, UA and OA (Fig. 1A), affect the viability of the human CRC cell line, HCT116. All three triterpenes reduced the viability of the HCT116 cells; however, the potency levels varied (Fig. 1B). CA was the most effective in inhibiting cell growth (with an $\mathrm{IC}_{50}$ of $24 \mu \mathrm{M}$ ), whereas UA and OA were less effective in reducing cell viability. The order of potency of these triterpenes was as follows; CA > UA > OA. These results suggest that $\mathrm{CA}$ is the most potent anti-proliferative agent among the three triterpenes examined.

CA induces morphological changes and chromatin condensation. Owing to the cytotoxic effects of $\mathrm{CA}$, we further investigated whether the growth inhibitory effects of CA are associated with the induction of apoptosis. Following expose to $\mathrm{CA}$ for $24 \mathrm{~h}$, the morphological changes of the cells were observed under a phase contrast light microscope. As shown in Fig. 2A (upper panel), CA altered the shape of the cells from a polygonal to a small round shape. The inhibition of cell growth was also detected in a concentration-dependent manner. In order to determine whether CA induces nuclear condensation, one of the characteristics of apoptosis, Hoechst staining was conducted. The untreated cells exhibited an intact nuclear structure, whereas nuclear condensation was observed in the cells treated with $\mathrm{CA}$ in a concentration-dependent manner (Fig. 2A, lower panel).

CA induces apoptosis in HCT116 cells. To confirm the effects of CA on apoptotic cell death, we performed an Annexin V staining-based flow cytometric analysis to assess the externalization of phosphatidylserine. Treatment with various concentrations of CA resulted in a concentration-dependent increase in both the early (Annexin $\mathrm{V}^{+} / \mathrm{PI}^{-}$) and late apoptotic cell population (Annexin $\mathrm{V}^{+} / \mathrm{PI}^{+}$) (Fig. 2B). The apoptotic indices were 5.6, 32.3, 58.1 and $68.9 \%$ at $0,10,20$ and $25 \mu \mathrm{M}$ $\mathrm{CA}$, respectively. Collectively, these results suggest that the growth inhibitory effects of CA were due, at least in part, to the apoptosis of HCT116 cells. We found that CA markedly increased the sub-G1 hypodiploid cell population (genomic DNA fragmentation) (Fig. 3). Treatment with CA at 10, 20 and $25 \mu \mathrm{M}$ for $24 \mathrm{~h}$ resulted in the accumulation of cells in the sub-G1 phase from $2.3 \%$ in the untreated control cells to 3.1, 14.2 and $32 \%$, respectively. Overall, these results indicate that the accumulation of the apoptotic population of colon cancer cells by CA may be responsible for the CA-induced inhibition of cell growth.

CA modulates the expression of apoptosis-related proteins. Our results thus far indicated that $\mathrm{CA}$ is capable of inducing apoptotic cell death. Therefore, we then investigated whether CA alters the levels of proteins involved in apoptosis in colon cancer cells. We first investigated whether CA affects the expression of pro-apoptotic markers in human CRC cells. The treatment of cells with CA upregulated the expression of p21, FasL, Fas and Bax in a concentration-dependent manner (Fig. 4A). However, the level of the pro-apoptotic protein, p53, was not altered significantly following treatment with CA, and it even decreased at the highest concentration of CA. This result suggests that CA induces apoptosis through a p53-independent mechanism. We then examined whether CA can modulate the expression of anti-apoptotic proteins. CA suppressed the expression of the anti-apoptotic proteins, Bcl-2 
A

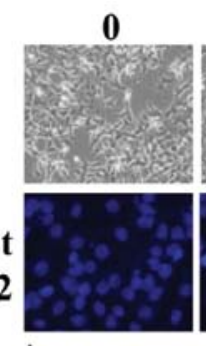

B

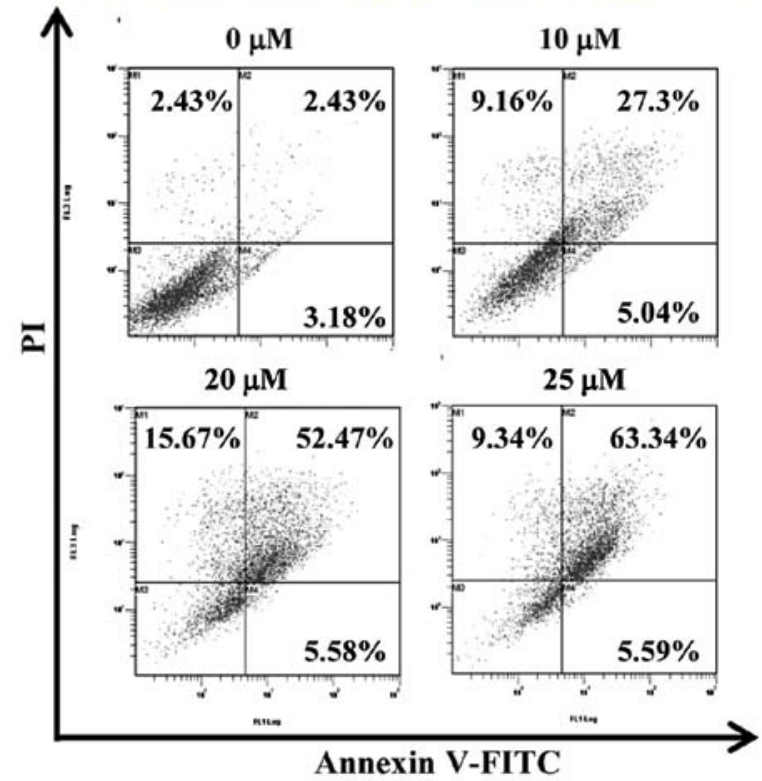

Figure 2. Effect of corosolic acid (CA) on the induction of apoptosis in HCT116 cells. (A) Cells were incubated in the presence of various concentrations of $\mathrm{CA}$ for $24 \mathrm{~h}$ and then stained with Hoechst 33342 for $20 \mathrm{~min}$. After staining, the cells were washed with phosphate-buffered saline (PBS) and photographed under a fluorescence microscope using a blue filter. Magnification, $\mathrm{x} 400$. Arrows indicate CA-induced chromatin condensation. (B) Cells were exposed to the indicated concentrations of $\mathrm{CA}$ for $24 \mathrm{~h}$, and double stained with Annexin V-FITC/propidium iodide (PI) and analyzed by flow cytometry. The data shown are representative of three independent experiments with similar results.

and survivin, in a concentration-dependent manner (Fig. 4B). The effects of $\mathrm{CA}$ on the expression of anti-apoptotic proteins was more prominent at 20 and $25 \mu \mathrm{M}$.

Activation of caspase-cascade is required for the induction of apoptosis by $C A$. Since the activation of the caspase pathway has been known to play a central role in the execution of apoptosis, we investigated whether the apoptotic cell death induced by $\mathrm{CA}$ was associated with the activation of caspases. First, we investigated whether CA activates caspases in HCT116 cells. The cells were treated with various concentrations of CA, and caspase activity was determined by a colorimetric protease assay. The results revealed a concentration-dependent increase in the activities of both initiator caspases (caspase-8 and -9) and effector caspases (caspase-3) (Fig. 5A). We also observed that CA markedly induced the activation of caspase- 3 in a concentration-dependent manner, while the activities of caspase- 8 and -9 were only slightly altered.

We then examined the effects of CA on the levels of caspase- $8,-9$ and -3 and poly(ADP-ribose) polymerase (PARP) by western blot analysis. Apoptosis is mediated through initi-

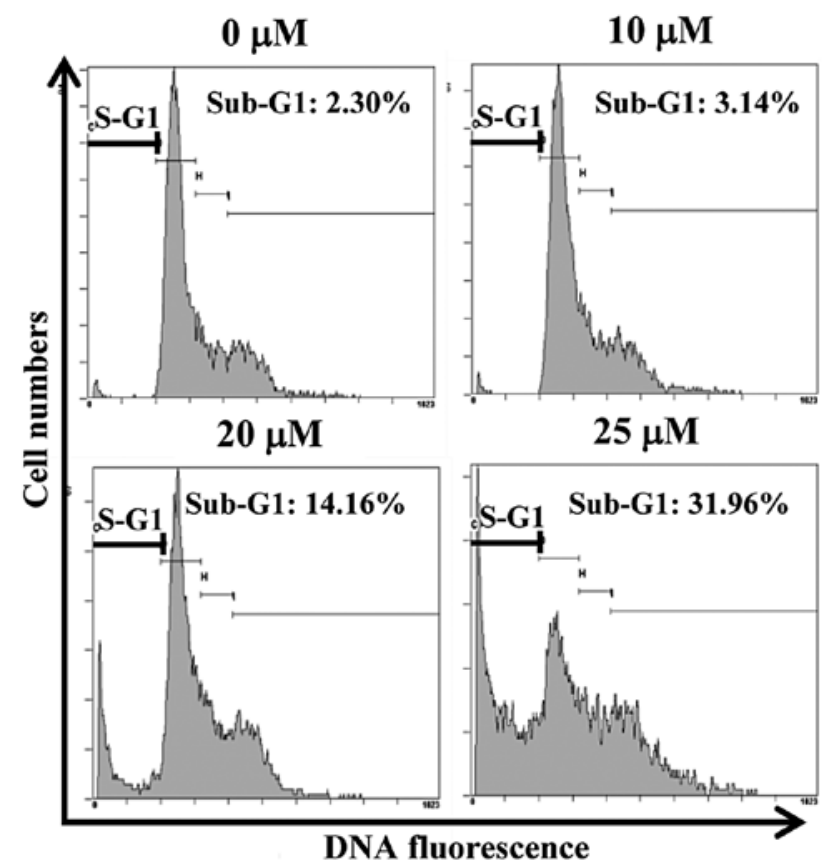

Figure 3. Effects of corosolic acid (CA) on the number of HCT116 cells in the sub-G1 phase. Cells were treated with the indicated concentrations of CA for $24 \mathrm{~h}$ and analyzed by flow cytometry following staining with propidium iodide (PI). The data shown are representative of three independent experiments with similar results. S-G1, sub-G1 phase.

ator caspases, which lead to caspase-3 activation that precedes the cleavage of PARP (5). CA induced a decrease in the levels of procaspase- $8,-9$ and -3 and the subsequent cleavage of PARP in HCT116 cells (Fig. 5B).

As the consecutive activation of caspases is necessary for apoptosis to take place and since CA activated caspases, we investigated whether the CA-induced apoptosis is caspase-dependent. The HCT116 cells were pre-treated with z-VAD-FMK, a pan-caspase inhibitor, prior to exposure of the cells to CA and then determined apoptosis by analyzing the cleavage of PARP. We found that CA induced the cleavage of PARP in a concentration-dependent manner. However, pre-treatment of the cells with Z-VAD-FMK abolished the CA-induced cleavage of PARP (Fig. 5C). Overall, these results suggest that the activation of caspases is involved in the induction of apoptosis by CA.

CA suppresses the expression of cell cycle regulatory proteins. It is well-known that the induction of apoptosis may be cell cycle-dependent (28). Thus, previous reports have indicated that CA induces G0/G1 arrest in human gastric cancer cells (24) and causes a late $\mathrm{S}$ phase or early G2/M phase block in cervical cancer cells (23). Therefore, it is possible that CA can cause apoptosis through the perturbation of the cell cycle. To examine this possibility, we investigated the effects of CA on the expression of the G1 regulators, cyclin D1 and cyclin $\mathrm{E}$, and of the G2/M regulator, cyclin $\mathrm{B}$, in HCT116 cells. CA suppressed the expression of cyclin D1, E and B1 in a concentration-dependent manner (Fig. 6). Collectively, these results indicate that the $\mathrm{CA}$-induced downregulation of cyclins is associated with apoptosis in colon cancer cells. 


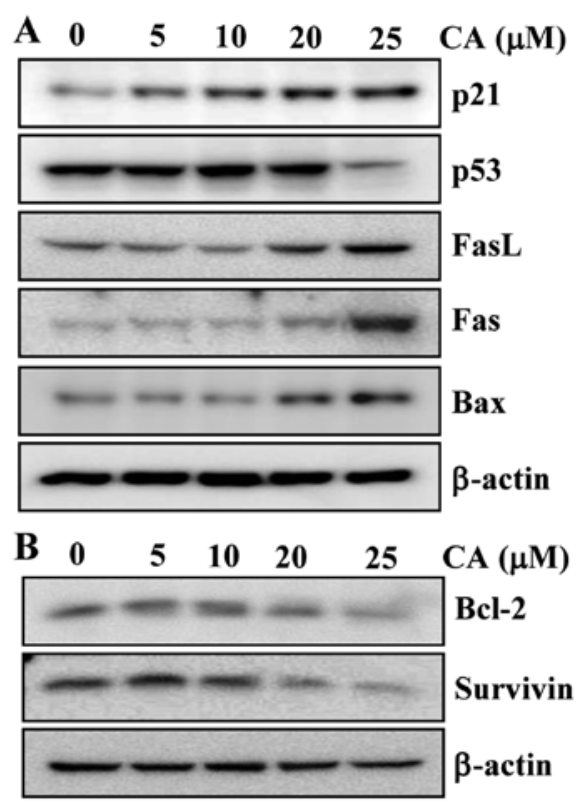

Figure 4. Effects of corosolic acid (CA) on pro-apoptotic and anti-apoptotic protein expression. Cells were incubated with various concentrations of CA for $24 \mathrm{~h}$. Cell lysates were prepared and immunoblotted. Western blot analysis was performed using relevant antibodies against (A) pro-apoptotic proteins and (B) anti-apoptotic proteins. $\beta$-actin was used as a protein loading control. Results are representative of one of three independent experiments with similar results.

\section{Discussion}

Worldwide, almost one million individuals develop CRC each year; thus, approximately $50 \%$ of these individuals can be expected to die due to systemic disease within five years of diagnosis (29). Although the incidence of CRC has been slowly declining over the past few decades, possibly due to improved screening and earlier detection methods, high mortality rates emphasize the desperate need for novel and improved therapies. In the present study, we aimed to determine whether the natural agent, CA, induces apoptosis in human CRC cells and to elucidate the possible mechanisms involved.

In present study, we compared the growth suppressive effects of three triterpenes in HCT116 cells. These included ursane types, such as CA and UA and oleanane types, such as OA (structures are illustrated in Fig. 1A). We found that the ursane-type triterpenes (CA and UA) had more potent inhibitory effects on cell viability than the oleanane type (OA) ones. In addition, CA was the most cytotoxic to HCT116 cells among the three tested compounds. In agreement with these observations, CA has been previously shown to exhibit the strongest anti-proliferative activity in four different human leukemia cell lines (9). In another study, CA exhibited cytotoxic effects in both C6 rat glioma and human epithelial carcinoma (A431) cells, whereas UA failed to show cytotoxic effects in A431 cells at the concentration of 10-100 $\mu \mathrm{M}(30)$. The authors suggested that the position and number of hydroxyls on triterpenes are critical to possess cytotoxicity, although the two agents harbor the same ursane-type pentacyclic triterpene cores. Of note, the only structural difference between UA and CA is a single additional hydroxyl group on the A-ring of the ursane-12-en
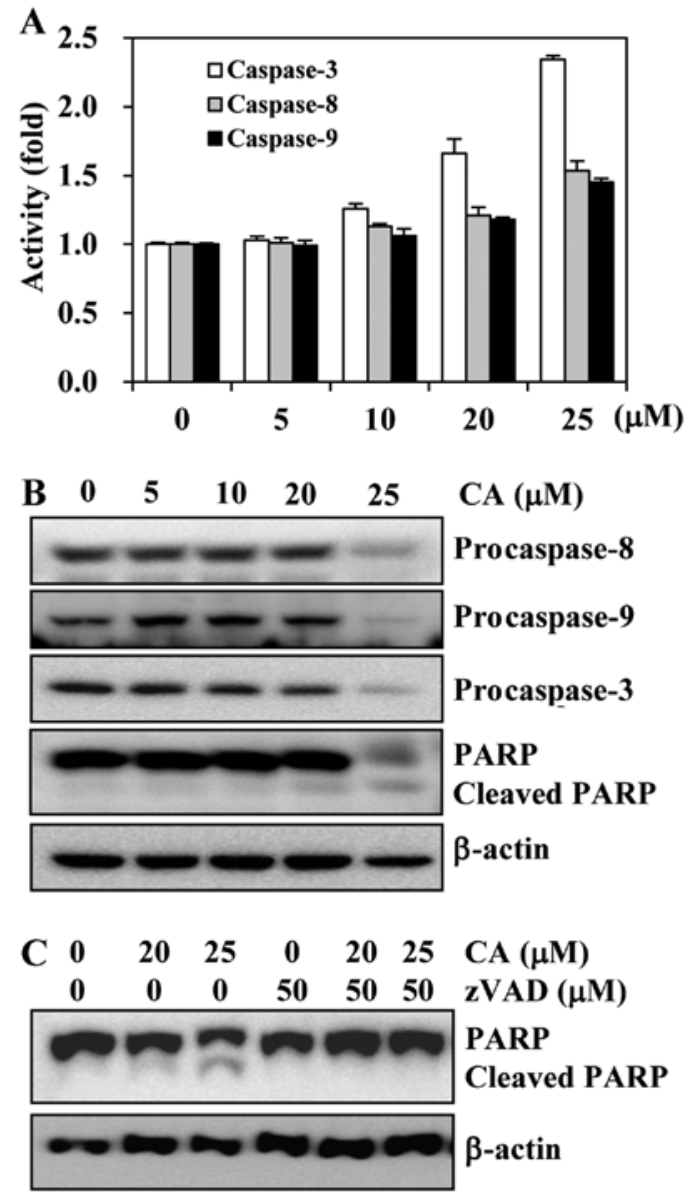

Figure 5. Caspase activation is involved in corosolic acid (CA)-induced apoptosis. (A) Cells were incubated with various concentrations of CA for $24 \mathrm{~h}$ Following incubation, cells were collected and caspase activity was assessed using p-nitroaniline (pNA)-labeled tetrapeptides as the substrate. Values are expressed as the means \pm standard deviation (SD) of duplicate samples. Results are representative of one of two independent experiments. (B) Cells were incubated with various concentrations of CA for $24 \mathrm{~h}$. Following incubation with CA, cell lysates were prepared and subjected to sodium dodecyl sulfatepolyacrylamide gel electrophoresis (SDS-PAGE). Western blot analysis was performed using relevant antibodies. $\beta$-actin was used as a protein loading control. Results are representative of one of three independent experiments with similar results. (C) HCT116 cells were pre-treated with $50 \mu \mathrm{M} z$-VADFMK (a broad caspase inhibitor) for $1 \mathrm{~h}$, then exposed to CA for $24 \mathrm{~h}$. Cell extracts were prepared and subjected to western blot analysis. $\beta$-actin was used as a protein loading control. Results are representative of one of three independent experiments with similar results.

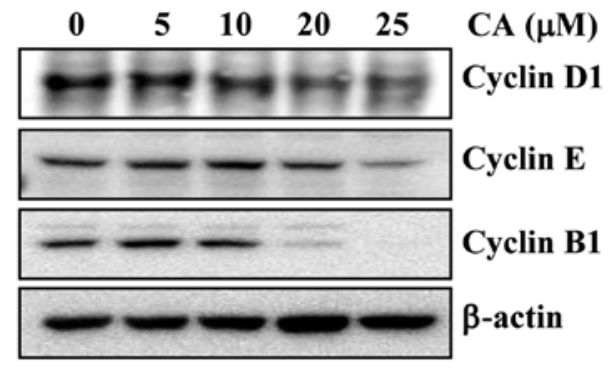

Figure 6. Effects of corosolic acid (CA) on cell cycle regulatory protein expression. Cells were exposed to various concentrations of CA for $24 \mathrm{~h}$, harvested and then lysed. Cell lysates were subjected to western blot analysis using relevant antibodies. $\beta$-actin was used as a protein loading control. Results are representative of one of three independent experiments with similar results. 
skeleton; thus, this difference is responsible for the selective cytotoxicity against $\mathrm{C} 6$ cells. Shifting the C-19 methyl to the C-20 position of CA, decreases the cytotoxic activity of CA (see OA in Fig. 1B). How and which position of the hydroxyl group of the A-ring in ursane-type triterpenes contributes to selective cytotoxicity remains unclear; thus, further analysis of the structure activity relationship is required.

The induction of apoptosis is considered a common event for anticancer agents. In principle, two major different activation pathways in apoptosis have been described; one involves the disruption of mitochondrial membrane potential and the release of cytochrome $c$. Released cytochrome $c$ binds to apoptotic protease activating factor 1 and then recruits procaspase- 9 , resulting in the formation of an apoptosome complex (known as the intrinsic pathway). The other pathway, also known as the extrinsic pathway, initiates with death receptor ligation or Fas/FasL interaction, resulting in the subsequent recruitment of Fas-associated death domain protein (FADD). FADD then associates with procaspase- 8 , resulting in the activation of caspase- 8 . Finally, both caspase- 9 and -8 activate caspase- 3 , leading to the execution of apoptosis $(31,32)$. In the present study, we found that CA induced the activity of caspase- $-3,-8$ and -9 , indicating that both the intrinsic and extrinsic pathways were activated. The observed caspase cascade-mediated apoptosis-inducing properties of CA are in agreement with those observed by others in osteosarcoma (21), leukemia (9), as well as cervical (23) and lung cancer cells (19). We also observed the CA-mediated cleavage of PARP, an indicator of caspase- 3 activation; this was abrogated when we employed the general caspase inhibitor, z-VAD-FMK. These findings provide evidence that the apoptosis induced by CA in HCT116 cells is mediated through caspase activation. However, which caspase is primarily involved in CA-induced apoptosis remains to be elucidated.

Other terpenoids, such as UA (33), saikosaponin D (34) and ginsenoside Rk1 (35), have been reported to induce apoptosis through the activation of Fas/FasL. Upon activation by the engagement with its ligand, FasL, Fas cleaves caspase- 8 and activates the caspase cascade, including caspase-3, leading to the activation of the extrinsic pathway (36). The downregulation of Fas occurs during tumor progression $(37,38)$, and many cancer cells obtain a survival strategy by decreasing their sensitivity to Fas-induced apoptosis (39). However, the role of FasL as a pro- or anti-apoptotic mediator has been largely controversial in colon cancer cells (40). In the present study, we found that CA upregulated the death receptor, Fas, and its ligand, FasL. In addition, the activation of caspase- 8 may have occurred due to the upregulation of Fas that may ultimately have sensitized the colon cancer cells to apoptosis by CA. Further research is required however, to determine the exact effects of CA on the Fas/FasL pathway. Our data suggest that CA induces apoptosis in HCT116 cells, at least in part, through the activation of the Fas/FasL pathway.

We found that the growth inhibitory effects of CA correlated with the downregulation of gene products, such as survivin and $\mathrm{Bcl}-2$, which are all known as anti-apoptotic proteins. Survivin is a member of the inhibitor of apoptosis proteins, the only endogenous proteins that inhibit the caspase cascade. The Bcl-2 family protein, Bcl-2, is also involved in the loss of caspase- 9 activation, and therefore provides a survival strategy to cancer cells from intrinsic pathway-mediated apoptosis. In agreement with our observations, the suppression of survivin and $\mathrm{Bcl}-2$ expression by $\mathrm{CA}$ has been shown in a previous study (19).

A recent study indicated that the generation of reactive oxygen species (ROS) by CA is a critical regulator of caspasemediated apoptosis in A549 lung cancer cells (19). Moreover, the exposure of cells to the ROS scavenger, $\mathrm{N}$-acetyl cysteine (NAC), prevented CA-induced cytotoxicity, as well as caspase activation (19). In the present study, NAC failed to prevent cell death induced by CA in the HCT116 cells (data not shown). The precise reason for this difference is not clear; however, the cell type and experimental conditions (i.e., dosage, time and analytical methods) used may account for this difference.

The majority of the anticancer properties assigned to CA have been demonstrated in in vitro studies. Although there are some reports on the anti-diabetic and antioxidant effects of CA in animals, only one animal study revealing the anticancer activities of CA has been reported. The oral administration of CA has been shown to be effective in animal models of murine osteosarcoma (20). The authors demonstrated that the development of tumors and lung metastasis in the animals was markedly suppressed by the oral administration of CA $(17.5 \mathrm{mg} / \mathrm{kg})$. To date, to the best of our knowledge, no animal studies have been conducted to assess the toxicity or $\mathrm{LD}_{50}$ values of CA; however, in this study, we provide strong evidence supporting the anticancer potential of CA.

Overall, the data from the present study provide evidence that CA induces apoptosis through the activation of caspases. This presents a rationale for the use of CA as an anticancer agent. However, further studies using clinically relevant animal models and human efficacy and safety studies are required to explore the therapeutic potential of CA against cancer.

\section{Acknowledgements}

This study was financially supported by the (2013 Post-Doc. Development Program) of Pusan National University, the National Research Foundation of Korea (NRF) Grant Funded by the Korea Government (MSIP) (no. 2009-0083538), and the R\&D Program of MKE/KEIT (10040391, Development of Functional Food Materials and Device for Prevention of Aging-associated Muscle Function Decrease). We thank the Aging Tissue Bank for providing research information.

\section{References}

1. Bray F, Jemal A, Grey N, Ferlay J and Forman D: Global cancer transitions according to the Human Development Index (2008-2030): a population-based study. Lancet Oncol 13: 790-801, 2012.

2. Jung KW, Won YJ, Kong HJ, Oh CM, Seo HG and Lee JS: Cancer statistics in Korea: incidence, mortality, survival and prevalence in 2010. Cancer Res Treat 45: 1-14, 2013.

3. Kanwar SS, Poolla A and Majumdar AP: Regulation of colon cancer recurrence and development of therapeutic strategies. World J Gastrointest Pathophysiol 3: 1-9, 2012.

4. Thompson CB: Apoptosis in the pathogenesis and treatment of disease. Science 267: 1456-1462, 1995.

5. Lowe SW and Lin AW: Apoptosis in cancer. Carcinogenesis 21: 485-495, 2000.

6. Newman DJ, Cragg GM and Snader KM: Natural products as sources of new drugs over the period 1981-2002. J Nat Prod 66: 1022-1037, 2003 
7. Ahn KS, Hahm MS, Park EJ, Lee HK and Kim IH: Corosolic acid isolated from the fruit of Crataegus pinnatifida var. psilosa is a protein kinase $\mathrm{C}$ inhibitor as well as a cytotoxic agent. Planta Med 64: 468-470, 1998

8. Li B, Meng X, Zhu L, Jiao X and Zhang J: Application of highspeed counter-current chromatography for isolation of triterpenes from Schisandra chinensis (Turcz.) Baill and induction apoptosis mechanism of HSC-T6. Biomed Mater Eng 24: 969-977, 2013.

9. Uto T, Sakamoto A, Tung NH, et al: Anti-proliferative activities and apoptosis induction by triterpenes derived from Eriobotrya japonica in human leukemia cell lines. Int J Mol Sci 14: 4106-4120, 2013.

10. Miura T, Takagi S and Ishida T: Management of diabetes and its complications with banaba (Lagerstroemia speciosa L.) and corosolic acid. Evid Based Complement Alternat Med 2012: 871495, 2012.

11. Caligiani A, Malavasi G, Palla G, Marseglia A, Tognolini M and Bruni R: A simple GC-MS method for the screening of betulinic, corosolic, maslinic, oleanolic and ursolic acid contents in commercial botanicals used as food supplement ingredients. Food Chem 136: 735-741, 2013.

12. Lee MS, Lee CM, Cha EY, et al: Activation of AMP-activated protein kinase on human gastric cancer cells by apoptosis induced by corosolic acid isolated from Weigela subsessilis. Phytother Res 24: 1857-1861, 2010.

13. Rao AR, Veeresham $C$ and Asres K: In vitro and in vivo inhibitory activities of four Indian medicinal plant extracts and their major components on rat aldose reductase and generation of advanced glycation endproducts. Phytother Res 27: 753-760, 2013.

14. Yin MC, Lin MC, Mong MC and Lin CY: Bioavailability, distribution, and antioxidative effects of selected triterpenes in mice. J Agric Food Chem 60: 7697-7701, 2012

15. Chen H, Yang J, Zhang Q, Chen LH and Wang Q: Corosolic acid ameliorates atherosclerosis in apolipoprotein E-deficient mice by regulating the nuclear factor- $\mathrm{\kappa B}$ signaling pathway and inhibiting monocyte chemoattractant protein-1 expression. Circ J 76: 995-1003, 2012.

16. Takagi S, Miura T, Ishihara E, Ishida T and Chinzei Y: Effect of corosolic acid on dietary hypercholesterolemia and hepatic steatosis in KK-Ay diabetic mice. Biomed Res 31: 213-218, 2010.

17. Klein G, Kim J, Himmeldirk K, Cao Y and Chen X: Antidiabetes and anti-obesity activity of Lagerstroemia speciosa. Evid Based Complement Alternat Med 4: 401-407, 2007.

18. Aguirre MC, Delporte C, Backhouse N, et al: Topical antiinflammatory activity of 2alpha-hydroxy pentacyclic triterpene acids from the leaves of Ugni molinae. Bioorg Med Chem 14: 5673-5677, 2006.

19. Nho KJ, Chun JM and Kim HK: Corosolic acid induces apoptotic cell death in human lung adenocarcinoma A549 cells in vitro. Food Chem Toxicol 56: 8-17, 2013.

20. Horlad H, Fujiwara Y, Takemura K, et al: Corosolic acid impairs tumor development and lung metastasis by inhibiting the immunosuppressive activity of myeloid-derived suppressor cells. Mol Nutr Food Res 57: 1046-1054, 2013.

21. Cai X, Zhang H, Tong D, et al: Corosolic acid triggers mitochondria and caspase-dependent apoptotic cell death in osteosarcoma MG-63 cells. Phytother Res: Feb 21, 2011 (Epub ahead of print).

22. Fujiwara Y, Komohara Y, Ikeda T and Takeya M: Corosolic acid inhibits glioblastoma cell proliferation by suppressing the activation of signal transducer and activator of transcription-3 and nuclear factor-kappa B in tumor cells and tumor-associated macrophages. Cancer Sci 102: 206-211, 2011.
23. Xu Y, Ge R, Du J, et al: Corosolic acid induces apoptosis through mitochondrial pathway and caspase activation in human cervix adenocarcinoma HeLa cells. Cancer Lett 284: 229-237, 2009.

24. Lee MS, Cha EY, Thuong PT, Kim JY, Ahn MS and Sul JY: Down-regulation of human epidermal growth factor receptor 2/neu oncogene by corosolic acid induces cell cycle arrest and apoptosis in NCI-N87 human gastric cancer cells. Biol Pharm Bull 33: 931-937, 2010.

25. Miura T, Itoh Y, Kaneko T, et al: Corosolic acid induces GLUT4 translocation in genetically type 2 diabetic mice. Biol Pharm Bull 27: 1103-1105, 2004.

26. Wen X, Xia J, Cheng K, et al: Pentacyclic triterpenes. Part 5: synthesis and SAR study of corosolic acid derivatives as inhibitors of glycogen phosphorylases. Bioorg Med Chem Lett 17: 5777-5782, 2007

27. Shi L, Zhang W, Zhou YY, et al: Corosolic acid stimulates glucose uptake via enhancing insulin receptor phosphorylation. Eur J Pharmacol 584: 21-29, 2008.

28. Vermeulen K, Berneman ZN and Van Bockstaele DR: Cell cycle and apoptosis. Cell Prolif 36: 165-175, 2003.

29. Weitz J, Koch M, Debus J, Höhler T, Galle PR and Buchler MW: Colorectal cancer. Lancet 365: 153-165, 2005.

30. Mazumder K, Tanaka K and Fukase K: Cytotoxic activity of ursolic acid derivatives obtained by isolation and oxidative derivatization. Molecules 18: 8929-8944, 2013.

31. Chang HY and Yang X: Proteases for cell suicide: functions and regulation of caspases. Microbiol Mol Biol Rev 64: 821-846, 2000.

32. Kischkel FC, Hellbardt S, Behrmann I, et al: Cytotoxicitydependent APO-1 (Fas/CD95)-associated proteins form a death-inducing signaling complex (DISC) with the receptor. EMBO J 14: 5579-5588, 1995.

33. Li Y, Xing D, Chen Q and Chen WR: Enhancement of chemotherapeutic agent-induced apoptosis by inhibition of NF-kappaB using ursolic acid. Int J Cancer 127: 462-473, 2010.

34. Hsu YL, Kuo PL and Lin CC: The proliferative inhibition and apoptotic mechanism of Saikosaponin D in human non-small cell lung cancer A549 cells. Life Sci 75: 1231-1242, 2004.

35. Kim JS, Joo EJ, Chun J, et al: Induction of apoptosis by ginsenoside Rk1 in SK-MEL-2-human melanoma. Arch Pharm Res 35: 717-722, 2012

36. Hengartner MO: The biochemistry of apoptosis. Nature 407: $770-776,2000$

37. Mizutani Y, Hongo F, Sato N, Ogawa O, Yoshida O and Miki T: Significance of serum soluble Fas ligand in patients with bladder carcinoma. Cancer 92: 287-293, 2001.

38. Osorio LM, Aguilar-Santelises M, De Santiago A, Hachiya T, Mellstedt $\mathrm{H}$ and Jondal M: Increased serum levels of soluble Fas in progressive B-CLL. Eur J Haematol 66: 342-346, 2001.

39. Khong HT and Restifo NP: Natural selection of tumor variants in the generation of 'tumor escape' phenotypes. Nat Immunol 3: 999-1005, 2002.

40. Zhu Q, Liu JY, Yang CM, et al: Influence of antitumor drugs on the expression of Fas system in SW480 colon cancer cells. Eur J Gastroenterol Hepatol 18: 1071-1077, 2006. 